\title{
Determination of trace levels of haloacetic acids and perchlorate in drinking water by ion chromatography with direct injection
}

\author{
Yongjian Liu, Shifen Mou* \\ Research Center for Eco-Environmental Sciences, Chinese Academy of Sciences, P.O. Box 2871, Beijing 100085, China
}

\begin{abstract}
Disinfection by products of haloacetic acids and perchlorate pose significant health risks, even at low $\mu \mathrm{g} / 1$ levels in drinking water. A new method for the simultaneous determination of nine haloacetic acids (HAAs) and perchlorate as well as some common anions in one run with ion chromatography was developed. The HAAs tested included mono-, di-, trichloroacetic acids, mono, di-, tribromoacetic acids, bromochloroacetic acid, dibromochloroacetic acid, and bromodichloroacetic acid. Two high-capacity anion-exchange columns, a carbonate-selective column and a hydroxide-selective hydrophilic one, were used for the investigation. With the carbonate-selective column, the nine HAAs as well as fluoride, chloride, nitrite, nitrate, phosphate and sulfate could be well separated and determined in one run. With the very hydrophilic column and a gradient elution of sodium hydroxide, methanol and deionized water, the nine HAAs, fluoride, chloride, nitrite, nitrate as well as perchlorate could be simultaneously determined in one run within 34 min. The detection limits for HAAs were between 1.11 and $9.32 \mu \mathrm{g} / 1$. For perchlorate, it was $0.60 \mu \mathrm{g} / 1$.
\end{abstract}

(C) 2003 Elsevier Science B.V. All rights reserved.

Keywords: Water analysis; Haloacetic acids; Perchlorate; Acetic acids; Inorganic anions

\section{Introduction}

The introduction of chlorination as a disinfection method for drinking water has dramatically reduced the number of deaths occurring annually from outbreaks of waterborne disease [1]. However, during the disinfection, some organohalogen species such as haloacetic acids (HAAs) which resulted from the reaction of hypochlorous acid with the humic and fulvic acids found naturally in water may be generated [2,3]. Toxicological studies indicate that these

\footnotetext{
*Corresponding author. Tel.: +86-10-6284-9182; fax: +86-106284-9239.

E-mail address: shifenm@mail.rcees.ac.cn (S. Mou).
}

compounds may have adverse effects on health and among others, dichloroacetic acid and trichloroacetic acid are animal carcinogens [4-6]. The United States Environmental Protection Agency (EPA) has introduced guidelines for the maximum contamination levels (MCLs) of the compounds. The stage 1 disinfection by-products (DBPs) rule for the MCLs of the sum of five HAAs [monochloro- (MCAA), monobromo- (MBAA), dichloro- (DCAA), dibromo(DBAA) and trichloroacetic acid (TCAA)] is set at $60 \mu \mathrm{g} / \mathrm{l}$. It is proposed that the level be lowered to $30 \mu \mathrm{g} / 1$ during upcoming stage 2 DBP negotiations.

Perchlorate originates as a contaminant in ground water and surface waters from the dissolution of ammonium, potassium, magnesium, or sodium salts. Sources for the contamination include chemical 
fertilizer and various other chemical and industrial sources. One major source of contamination is the manufacture of ammonium perchlorate for use as the oxidizer component and primary ingredient in solid propellant for rockets, missiles, and fireworks. Perchlorate is also used on a large scale as a component of air bag inflators [7]. It has recently been found in drinking water wells in areas where aerospace materials and munitions have been manufactured and tested [8]. Perchlorate is a health concern, as it interferes with the ability of the thyroid gland to produce thyroid hormones. Therefore, it has been added to the EPA's unregulated contaminant monitoring list [9-12]. In California, the Department of Health Services issued an action level of $18 \mu \mathrm{g} / 1$ perchlorate in California drinking water supplies [13].

Presently, the mainly practical methods for the determination of HAAs in water are gas chromatography (GC) and mass spectrometry (MS) based methods, high-performance liquid chromatography (HPLC), capillary electrophoresis (CE) and ion chromatography (IC). GC is the most widely used technique $[14,15]$. However, the method suffers from several drawbacks, such as the labor-intensive extraction procedure and toxic derivatization reagents, etc. MS-based methods for the determination of HAAs in water have been developed recently [1618]. Though the MS-based detection techniques can be used to achieve good detection limits, they each added considerable complexity and significant cost to the analytical method. In addition, coelutions of some HAAs during the determination were not well resolved in some cases [19]. HPLC can also be used for the determination of HAAs, yet the detection limits are not satisfactory [20]. Recently, the application of CE to the detection of HAAs has become increasingly popular [21,22]. Although CE does not require the derivatization step and can reduce analysis time, the detection limits were unsatisfactory, and recoveries of some analytes were poor. HAAs can also be detected with anion-exchange IC in the conductivity mode [23-26]. Several IC methods with different anion-exchange columns were used for the determination of HAAs. However, some of the methods' detection limits were not sensitive, other methods suffered the complicated procedure of sam- ple pretreatment. Even though good results could be obtained, interference could be easily introduced into the sample. Therefore, a convenient and sensitive method for the direct determination of the HAAs is highly desirable.

The detection of perchlorate in water mainly concentrated on MS-based techniques [27,28] and IC methods [29-31]. Despite the good detection limit, the MS-based technique is a little complex, and the cost is significant. A modified carbon-paste electrode could also be used for the determination of perchlorate in drinking water. However, the detection limit was unsatisfactory [32]. With IC, not only can a comparable detection limit for perchlorate be achieved, but the method is also easy to handle.

The aim of our work is to study the simultaneous determination of nine HAAs and perchlorate in one injection with IC. Two high-capacity columns were used for the study. One is a carbonate-selective column. The other is a highly hydrophilic, hydroxide-selective column. The developed methods were used for the determination of HAAs and perchlorate in real drinking water samples.

\section{Experimental}

\subsection{Instrumentation}

A Dionex Model DX-500 ion chromatograph (Sunnyvale, CA, USA) equipped with a GP40 gradient pump, a Dionex IonPac AS16 analytical column $(250 \times 4 \mathrm{~mm})$ and an IonPac AG16 guard column $(50 \times 4 \mathrm{~mm})$, an IonPac AS9HC analytical column $(250 \times 4 \mathrm{~mm})$ and an IonPac AG9HC guard column $(50 \times 4 \mathrm{~mm})$ were used throughout. All the detection was performed by a Dionex ED40 electrochemical detector in the conductivity mode. Suppression of eluent for the AS9HC column was achieved by a Dionex ASRS-ULTRA (4 mm) suppressor and anion Atlas electrolytic suppressor, respectively. The Atlas suppressor was controlled with a Dionex SC20 suppressor controller. Both suppressors were run in the autosuppression recycle mode. For the AS16 column, the suppressor was operated in the autosuppression external water mode. The regenerant was deionized water, and the flow-rate was kept at 
$5.0 \mathrm{ml} / \mathrm{min}$. A $500-\mu \mathrm{l}$ sample loop was used for the injection. The Dionex OnGuard II Ag cartridge $(1 \mathrm{ml})$ was used for the removal of chloride in the samples. All tubing in the chromatography path (from the outlet of the pump to the exit of the suppressor) was polyether ether ketone (PEEK) [0.005 in. (0.125 mm) I.D.]. Both instrument control and data collection were performed with a personal computer and Dionex PeakNet 5.11 chromatography workstation.

\subsection{Chemicals}

All chemicals were of analytical-reagent grade unless specified. Monochloroacetic acid (MCAA), dichloroacetic acid (DCAA), trichloroacetic acid (TCAA), monobromoacetic acid (MBAA), dibromoacetic acid (DBAA), tribromoacetic acid (TBAA), bromochloroacetic acid (BCAA), bromodichloroaceitc acid (BDCAA), dibromochloroacetic acid (DBCAA) and perchlorate were purchased from Chem Service (USA). The methanol used was of chromatographical grade. The standard solution $(1000 \mathrm{mg} / \mathrm{l})$ of fluoride, chloride, nitrite, nitrate, phosphate and sulfate was purchased from National Research Center of Standard Reference Material (Beijing, China). All reagents, eluent and standard solutions were prepared using deionized water further purified with a EASYpure LF system (Barnstead) with a specific resistance of $18.3 \mathrm{M} \Omega \mathrm{cm}$, filtered through a $0.2 \mu \mathrm{m}$ membrane filter. The stock solutions of perchlorate and nine HAAs except for DCAA $(1000 \mathrm{mg} / \mathrm{l})$ were prepared by dissolving the corresponding mass of the dried salts in purified water. The stock solution of DCAA was prepared with the 99\% DCAA solution. All the solutions were stored in a refrigerator at $4{ }^{\circ} \mathrm{C}$ and they were stable for at least 2 weeks. Working-standard solutions were prepared daily by serial dilution of the stock solution prior to use. For the AS16 column, the eluent was $100 \mathrm{~m} M \mathrm{NaOH}$, deionized water and $10 \%$ aqueous methanol solution. The $\mathrm{NaOH}$ eluent was prepared with $50 \%(\mathrm{w} / \mathrm{w})$ sodium hydroxide solution. For the AS9HC column, the eluents were $28 \mathrm{mM} \mathrm{Na} \mathrm{CO}_{3}$ and $11.5 \mathrm{mM} \mathrm{Na} \mathrm{CO}_{3}$ for the different suppressors, respectively. All the flow-rates were kept at $1.0 \mathrm{ml} /$ min. All the determinations were performed in room temperature. Each sample was injected in triplicate.

\section{Results and discussion}

\subsection{Analysis of HAAs and perchlorate with the high-capacity carbonate-selective column}

\subsubsection{Separation of the analytes}

In the analytes, fluoride, MCAA, MBAA and chloride are weakly retained anions. In real drinking water sample, the concentration of chloride will be very high. If the retention times of fluoride, MCAA and MBAA are close to that of chloride, the high response of chloride will seriously interfere the detection of the other three anions. In order to separate the four anions well, a high-capacity column on which the anions could be tightly bound was preferred.

The IonPac AS9HC column is an anion-exchange

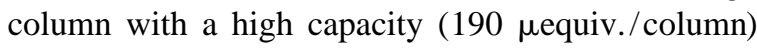
and can be used for the separation of fluoride, MBAA, MCAA with high concentration of chloride. With the column, a high ionic strength eluent should be used to achieve satisfactory results. Carbonate has strong affinity with stationary phase of the column. With a high concentration of carbonate, strongly retained anions can be quickly eluted. Considering the separation of the weakly retained anions together, a moderate concentration $(28 \mathrm{mM})$ of sodium carbonate was used as eluent thoroughly. With the eluent, nine HAAs and six common anions except for perchlorate could be well separated and determined simultaneously within $41 \mathrm{~min}$ in one run while the flow-rate was kept at $1.0 \mathrm{ml} / \mathrm{min}$. For the weakly-bound compounds, the concentration of the eluent was so strong that they were eluted quickly. Though the concentration of chloride in real sample was much higher than that of MBAA, it did not interfere the determination of MBAA. With respect to perchlorate, it is a highly polarizable anion and has very strong retention on the column. It cannot be eluted from the column under this condition.

For the tightly bound HAAs (TCAA, DBCAA, BDCAA and TBAA), they were difficult to be elute, which resulted in a long analysis procedure. The 
responses of the four compounds were very low, and the detection limits were poor. In order to improve the detection limits of the four analytes, a flow-rate gradient should be used to precipitate the elution. After the elution of sulfate at $15.0 \mathrm{~min}$, the flow-rate was enhanced from 1.0 to $1.5 \mathrm{ml} / \mathrm{min}$ within $0.1 \mathrm{~min}$ and kept for $20 \mathrm{~min}$. Then the flow-rate was decreased to $1.0 \mathrm{ml} / \mathrm{min}$ at $35.2 \mathrm{~min}$. With the flow-rate gradient, the retention time of the last compound (TBAA) was shortened by about $6 \mathrm{~min}$, the responses of the last four compounds were much heightened and the detection limits were greatly
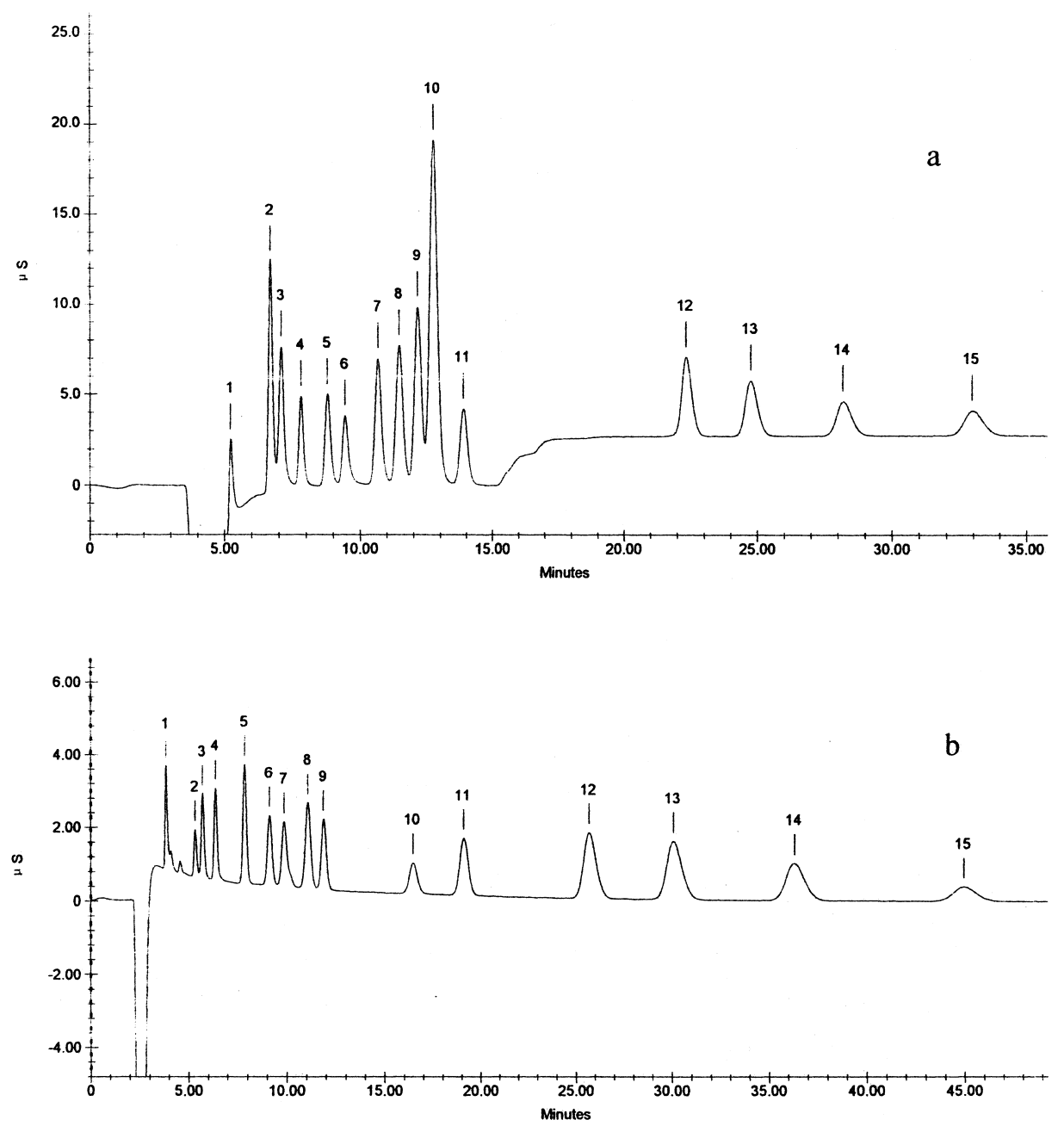

Fig. 1. The chromatogram of the standard solution of nine HAAs and six common anions on the AS9HC column suppressed with the ASRS-Ultra suppressor and anion Atlas electrolytic suppressor. (a) Dionex IonPac AG9HC and AS9HC columns; detection: suppressed conductivity with ASRS-ULTRA $4 \mathrm{~mm}$ operated at $300 \mathrm{~mA}$ in the autosuppression recycle mode; injection volume: $500 \mu \mathrm{l}$; eluent: $28 \mathrm{~m} M$ $\mathrm{Na}_{2} \mathrm{CO}_{3}$; flow-rate: $1.0 \mathrm{ml} / \mathrm{min}$ from $0 \mathrm{~min}$ to $15 \mathrm{~min}, 1.5 \mathrm{ml} / \mathrm{min}$ from $15.1 \mathrm{~min}$ to $35 \mathrm{~min}, 1.0 \mathrm{ml} / \mathrm{min}$ from $35.1 \mathrm{~min} .1=$ Fluoride $(0.15$ $\mathrm{mg} / \mathrm{l}), 2=\operatorname{MCAA}(2 \mathrm{mg} / 1), 3=\operatorname{MBAA}(2 \mathrm{mg} / 1), 4=$ chloride $(0.15 \mathrm{mg} / 1), 5=$ nitrite $(0.5 \mathrm{mg} / 1), 6=\mathrm{DCAA}(2 \mathrm{mg} / 1), 7=\mathrm{BCAA}(2 \mathrm{mg} / 1)$, $8=$ nitrate $(0.5 \mathrm{mg} / 1), 9=\operatorname{DBAA}(8 \mathrm{mg} / 1), 10=$ phosphate $(0.8 \mathrm{mg} / \mathrm{l}), 11=$ sulfate $(0.8 \mathrm{mg} / 1), 12=\mathrm{TCAA}(4.5 \mathrm{mg} / 1), 13=\mathrm{BDCAA}(5$ $\mathrm{mg} / 1), 14=\operatorname{DBCAA}(5 \mathrm{mg} / 1), 15=$ TBAA $(5 \mathrm{mg} / 1)$. (b) Dionex IonPac AG9HC and AS9HC columns; detection: suppressed conductivity with anion Atlas electrolytic suppressor $(4 \mathrm{~mm})$ operated at $80 \mathrm{~mA}$ in the autosuppression recycle mode; injection volume: $500 \mu \mathrm{l}$; eluent: $11.5 \mathrm{mM} \mathrm{Na} \mathrm{CO}_{3}$; flow-rate: $1.0 \mathrm{ml} / \mathrm{min} .1=$ Fluoride $(0.1 \mathrm{mg} / 1), 2=\mathrm{MCAA}(0.3 \mathrm{mg} / 1), 3=\mathrm{MBAA}(1 \mathrm{mg} / 1), 4=\mathrm{chloride}(0.15 \mathrm{mg} / 1)$, $5=$ nitrite $(0.5 \mathrm{mg} / 1), 6=$ DCAA $(1 \mathrm{mg} / 1), 7=$ BCAA $(1 \mathrm{mg} / 1), 8=$ nitrate $(0.5 \mathrm{mg} / 1), 9=$ DBAA $(3 \mathrm{mg} / 1), 10=$ phosphate $(0.75 \mathrm{mg} / 1)$, $11=$ sulfate $(0.75 \mathrm{mg} / 1), 12=$ TCAA $(5 \mathrm{mg} / 1), 13=$ BDCAA $(10 \mathrm{mg} / 1), 14=$ DBCAA $(10 \mathrm{mg} / 1), 15=$ TBAA $(10 \mathrm{mg} / 1)$. 
improved. All the analytes could be determined in 35 min (Fig. 1a). With regard to the change of the flow-rate, it only resulted in about a $2.8-\mu$ s increment of the background conductivity, which did not influence the determination of the analytes. In addition, the flow-rate gradient was very easy to handle. The whole IC system only needs 2 min to reach the new equilibrium, and then the next injection could be run immediately. By contrast with the eluent gradient, the flow-rate gradient is convenient and timesaving. Moreover, it does not need a gradient pump which costs significantly.

When carbonate was used as eluent, one of the major problems was the high baseline noise, which restricted the detection limits of analytes. The anion electrolytic suppressor (Atlas) is continuous electrolytically regenerated suppressor, which can offer much improved performance in baseline noise. In this investigation, the suppressor was used to determine of 15 compounds. It could be seen that all the analytes were well separated with $11.5 \mathrm{~m} M$ sodium carbonate (Fig. 1b). Compared with the chromatogram obtained with the ASRS-Ultra suppressor, due to the relatively small internal volume, the water dip was eluted out much more quickly. The peak of fluoride was far away from the dip and could be accurately detected. Because the strength of the eluent is a little weak, the whole procedure lasted for $47 \mathrm{~min}$. Though a flow-rate gradient could also be used, the baseline noise would be significantly increased, and baseline drift would occur. The detection limits of the tightly bound compounds would not be improved. Consequently, the isocratic elution was the best choice for the nine HAAs with the suppressor.

\subsubsection{Detection limits and linear ranges}

Large-volume injection proved a convenient method to enhance the sensitivity of analytes [33]. Due to the high capacity of the column, a large volume of $500 \mu \mathrm{l}$ sample loop was preferred. The detection limits of the analytes achieved with the column and suppressors are listed in Table 2. It could be seen that with the ASRS-Ultra suppressor, the detection limits of the nine HAAs were between 1.53 and $53.60 \mu \mathrm{g} / \mathrm{l}$. With the Atlas suppressor, the results were between 0.37 and $31.64 \mu \mathrm{g} / 1$.

\subsection{Analysis of HAAs and perchlorate with the} highly hydrophilic column

\subsubsection{Selection and optimization of eluent}

TBAA and perchlorate are hydrophobic anions. They have strong affinity to the stationary phase of column. In order to elute the two compounds quickly, a hydrophilic column should be chosen. The IonPac AS16 column is an extremely hydrophilic, high-capacity and hydroxide-selective anion-exchange column. With the column, the hydrophobic anions such as TBAA and perchlorate can be easily eluted with a strong hydroxide eluent. In order to separate the weakly retained anions such as fluoride and MCAA, a weak eluent should be used. Therefore, a gradient of sodium hydroxide and deionized water was preferred. In the analyzed compounds, chloride and MBAA, nitrate and DCAA have almost the same affinity on the column. It was very difficult to effectively separate the two groups of compounds only with the gradient of sodium hydroxide and deionized water.

Solvent can be added to ionic eluent to improve separation of anions, especially for those that have different hydrophobicities by altering the retention of the compounds. The hydrophobicities of MBAA and DCAA are slightly higher than those of chloride and nitrate, respectively. A solvent that can extend the retention of analytes should be used to improve the resolution of the two pairs of analytes.

Typical solvents used in IC are acetonitrile, methanol and isopropanol. Among the three solvents, acetonitrile was usually used to decrease the retention of the analytes on column [34]. It was not suitable for improving the resolution of the two groups of compounds. At low concentrations, isopropanol had a similar property to methanol in prolonging the retention of analytes on column, the high-purity methanol is much easier to obtain and is much cheaper than isopropanol. In this study, methanol was chosen as solvent. In addition, in order to prevent the volatilization of methanol, a watermethanol mixture was preferred.

The use of methanol as solvent will cause the variation of viscosity of the mixture and generate back pressure that is heavily influenced by the methanol content in the water-methanol mixture. If the methanol content is too high, the generated high 


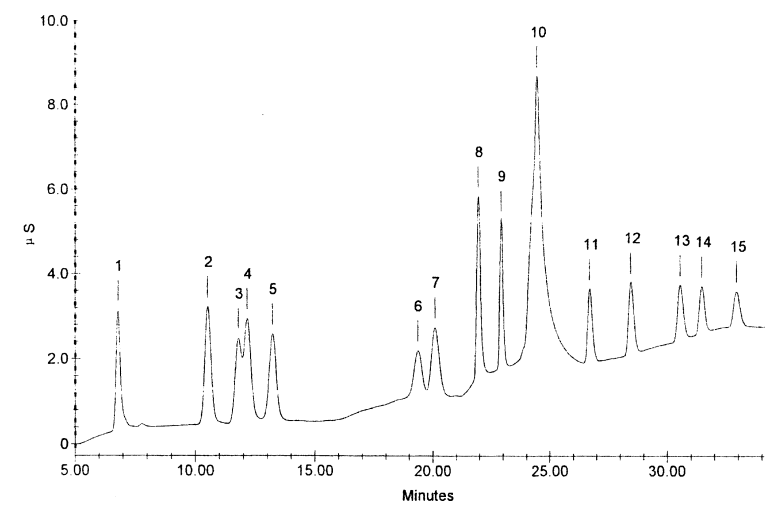

Fig. 2. The chromatogram of the standard solution of nine HAAs, perchlorate and four common anions. Dionex IonPac AG16 and AS16 columns; detection: suppressed conductivity with ASRSULTRA $4 \mathrm{~mm}$ operated at $300 \mathrm{~mA}$ in the external water mode; injection volume: $500 \mu \mathrm{l}$; eluent: $\mathrm{NaOH}$ in gradient, $4.9 \mathrm{~m} M$ for $9 \mathrm{~min}, 4.9-5.2 \mathrm{~m} M$ in $3 \mathrm{~min}, 5.2-7.2 \mathrm{mM}$ in $5 \mathrm{~min}, 7.2-30.9 \mathrm{mM}$ in $3 \mathrm{~min}, 30.9-72.5 \mathrm{mM}$ in $9 \mathrm{~min}, 72.5 \mathrm{mM}$ for $4 \mathrm{~min}$, followed by a linear gradient to $4.9 \mathrm{mM}$ in $7 \mathrm{~min}$, the content of $10 \%$ methanol solution was $5 \%$ in the eluent all along; flow-rate: $1.0 \mathrm{ml} / \mathrm{min} . \quad 1=$ Fluoride $(0.1 \mathrm{mg} / \mathrm{l}), 2=\mathrm{MCAA}(1 \mathrm{mg} / 1), 3=$ chloride $(0.1 \mathrm{mg} / 1), 4=\mathrm{MBAA}(2 \mathrm{mg} / 1), 5=$ nitrite $(0.5 \mathrm{mg} / \mathrm{l})$, $6=$ nitrate $(0.6 \mathrm{mg} / 1), 7=$ DCAA $(1 \mathrm{mg} / 1), 8=$ BCAA $(1 \mathrm{mg} / 1)$, $9=$ DBAA $(1.6 \mathrm{mg} / \mathrm{l}), 10=$ system peak, $11=$ TCAA $(1 \mathrm{mg} / \mathrm{l})$, $12=$ BDCAA $(2 \mathrm{mg} / 1), 13=$ DBCAA $(2 \mathrm{mg} / 1), 14=$ perchlorate $(0.2 \mathrm{mg} / 1), 15=$ TBAA $(2 \mathrm{mg} / 1)$.

back pressure will cause the leakage of the IC system. On the contrary, it will make no improvement on the retention of the analytes. By fixing the ratio of the methanol-water mixture in the eluent, a series of experiments were done by changing the content of methanol in the mixture. The result indicated that $10 \%$ aqueous methanol solution was the best choice.

The effect of $10 \%$ methanol in the methanolwater eluent on the resolution of the two pairs of analytes was investigated with the standard solution.
It was found that the peak resolution of MBAA and chloride reached the maximum $\left(R_{s}=0.95\right)$ [35] when the methanol-water $(10: 90, \mathrm{v} / \mathrm{v})$ mixture in the eluent was 5\%. MBAA and chloride could be quantitated. For DCAA and nitrate, the resolution was 1.14 . When the methanol-water solution accounted for $10 \%$ methanol, the optimal resolution $\left(R_{\mathrm{s}}=1.22\right)$ could be obtained for DCAA and nitrate. However, the back pressure caused by the solution was so high that it could lead to leakage of the system. Moreover, the resolution of MBAA and chloride was very poor $\left(R_{\mathrm{s}}=0.58\right)$. Considering the simultaneous separation and quantitation of the two groups of compounds, the methanol content in the methanol-water eluent was set at 5\%. From Fig. 2, it could be seen that nine HAAs, fluoride, chloride, nitrite, nitrate as well as perchlorate could be separated and quantitated with the selected eluent in gradient. With regard to sulfate and phosphate in sample, sulfate was co-eluted with the system peak, phosphate could be completely removed with OnGuard Ag cartridge. Neither of them interfered with the detection of HAAs.

Due to the difference in the functional group between the AS16 and AS9-HC columns, the retention of some compounds on the column was changed. On the AS9-HC column, chloride, nitrate and perchlorate were eluted after MBAA, DCAA and TBAA, respectively. On the AS16 column, chloride, nitrate and perchlorate were eluted before the corresponding anions, respectively.

\subsubsection{Sample treatment}

In real drinking water samples, the concentration of chloride is much higher than that of MBAA. The big response of chloride may cover up the small peak of MBAA. Considering the accurate determination of MBAA, chloride should be removed prior to injection into the column. The OnGuard Ag cartridge

Table 1

The recoveries of the 12 analytes after treatment with OnGuard II Ag cartridge

\begin{tabular}{|c|c|c|c|c|c|c|c|c|c|c|c|c|}
\hline & Fluoride & MCAA & MBAA & DCAA & Nitrate & BCAA & DBAA & TCAA & BDCAA & DBCAA & Perchlorate & TBAA \\
\hline Concentration (mg/l) & 0.15 & 1 & 2 & 1 & 0.5 & 1 & 1.6 & 1 & 2 & 2 & 2 & 2 \\
\hline Average recovery $(n=7, \%)$ & 98.8 & 99.1 & 98.5 & 97.9 & 96.8 & 98.0 & 97.1 & 96.9 & 96.8 & 96.6 & 95.9 & 97.7 \\
\hline $\operatorname{RSD}(n=7, \%)$ & 3.16 & 2.48 & 2.39 & 3.05 & 2.40 & 2.99 & 3.18 & 3.22 & 3.07 & 2.59 & 3.27 & 2.73 \\
\hline
\end{tabular}


Table 2

The detection limits and linear concentration range for the analytes

\begin{tabular}{|c|c|c|c|c|c|c|c|c|c|c|c|c|c|c|c|c|}
\hline & Fluoride & MCAA & MBAA & Chloride & Nitrite & DCAA & BCAA & DBAA & Nitrate & Phosphate & Sulfate & TCAA & BDCAA & DBCAA & TBAA & Perchlorate \\
\hline \multicolumn{17}{|l|}{ AS9HC-ASRS-Ultra } \\
\hline Concentration $(\mu \mathrm{g} / \mathrm{l})$ & 150 & 300 & 300 & 150 & 300 & 500 & 500 & 500 & 300 & 300 & 300 & 500 & 500 & 500 & 500 & \\
\hline $\operatorname{RSD}(n=7, \%)$ & 6.3 & 5.4 & 4.1 & 5.9 & 3.4 & 3.1 & 7.8 & 8.1 & 5.5 & 6.6 & 5.3 & 3.6 & 2.9 & 2.8 & 2.4 & \\
\hline Concentration range $(\mu \mathrm{g} / \mathrm{l})$ & $1-500$ & $2-50$ & $5-50$ & $1-1000$ & $2-500$ & $5-100$ & $5-50$ & $15-50$ & $2-100$ & $1-500$ & $4-1000$ & $5-50$ & $30-100$ & $40-200$ & $60-200$ & \\
\hline Correlation coefficient $(r)$ & 0.9801 & 0.9898 & 0.9901 & 0.9876 & 0.9994 & 0.9989 & 0.9988 & 0.9986 & 0.9887 & 0.9881 & 0.9969 & 0.9992 & 0.9989 & 0.9991 & 0.9990 & \\
\hline \multicolumn{17}{|l|}{ AS9HC-Atlas } \\
\hline $\operatorname{RSD}(n=7, \%)$ & 3.2 & 2.7 & 2.6 & 3.1 & 2.1 & 1.9 & 2.3 & 3.5 & 1.8 & 2.4 & 2.5 & 1.6 & 1.8 & 2.4 & 2.0 & \\
\hline Detection limit $(\mu \mathrm{g} / \mathrm{l})$ & 0.09 & 0.37 & 0.58 & 0.15 & 0.38 & 0.78 & 0.69 & 1.75 & 0.64 & 1.98 & 1.09 & 2.16 & 8.17 & 12.87 & 31.64 & \\
\hline Concentration range $(\mu \mathrm{g} / \mathrm{l})$ & $0.2-500$ & $1-50$ & $2-50$ & $1-1000$ & $1-500$ & $2-200$ & $2-100$ & $5-100$ & $1-500$ & $3-500$ & $5-5000$ & $8-100$ & $15-150$ & $20-200$ & $50-200$ & \\
\hline Correlation coefficient $(r)$ & 0.9993 & 0.9992 & 0.9988 & 0.9996 & 0.9989 & 0.9990 & 0.9986 & 0.9886 & 0.9981 & 0.9991 & 0.9992 & 0.9993 & 0.9991 & 0.9884 & 0.9889 & \\
\hline \multicolumn{17}{|l|}{ AS16 } \\
\hline Concentration $(\mu \mathrm{g} / \mathrm{l})$ & 100 & 300 & 300 & 100 & 300 & 500 & 500 & 500 & 300 & & & 500 & 500 & 500 & 500 & 500 \\
\hline $\operatorname{RSD}(n=7, \%)$ & 7.8 & 3.4 & 8.1 & 8.6 & 4.3 & 5.9 & 4.3 & 3.6 & 6.8 & & & 2.9 & 2.5 & 3.0 & 2.3 & 2.4 \\
\hline Detection limit* $(\mu \mathrm{g} / \mathrm{l})$ & 0.29 & 1.46 & 2.49 & 0.31 & 0.89 & 2.16 & 1.11 & 2.18 & 0.67 & & & 1.85 & 4.07 & 5.55 & 9.32 & 0.60 \\
\hline Correlation coefficient $(r)$ & 0.9881 & 0.9991 & 0.9815 & 0.9867 & 0.9987 & 0.9905 & 0.9986 & 0.9994 & 0.9905 & & & 0.9990 & 0.9991 & 0.9989 & 0.9995 & 0.9996 \\
\hline
\end{tabular}

* Calculated as three times the noise. 
containing high-capacity strong-acid cation-exchange resin in the silver form is used to remove chloride, bromide and phosphate, etc., by precipitation. The cartridge is especially effective for the removal of chloride. Even though the concentration of chloride in the sample is up to $400 \mathrm{mg} / \mathrm{l}$, more than $99.9 \%$ of chloride can be removed with one cartridge [36]. In this study, with the removal of chloride, MBAA could be accurately determined. Its recovery was up to $98.5 \%$. For other analytes, excellent recoveries could also be got except for nitrite (Table 1). After treatment, almost all the nitrite was removed with the cartridge. This was because in acidic solution, nitrite is heavily adsorbed by the cartridge. The mixed standard solution of the 16 analytes was a weakly acidic solution ( $\mathrm{pH}$ 5.9), which resulted in the poor recovery of nitrite.

\subsubsection{Detection limits and linear ranges}

The initial concentration of the eluent was very weak, the weakly retained anions could be held for a long time and far away from the water dip. In addition, due to the high capacity of the AS16 column, the introduction of a relatively large sample volume was preferred. In this experiment, a 500- $\mu 1$ sample loop was used for sample analysis. All the detection limits and linear ranges of the analytes are listed in Table 2. It can be seen that the detection limits for the nine HAAs were between 1.11 and $9.32 \mu \mathrm{g} / 1$. For perchlorate, the detection limit was $0.6 \mu \mathrm{g} / 1$.

The achieved results were compared with those obtained with the AS9HC column. It was found that for the weakly retained HAAs (MCAA, MBAA, DCAA, BCAA and DBAA), the detection limits acquired with the AS9HC column and Atlas suppressor were the best among the three methods. For the tightly bound HAAs (TCAA, BDCAA, DBCAA, TBAA), the results obtained with the AS16 column were the optimum values.
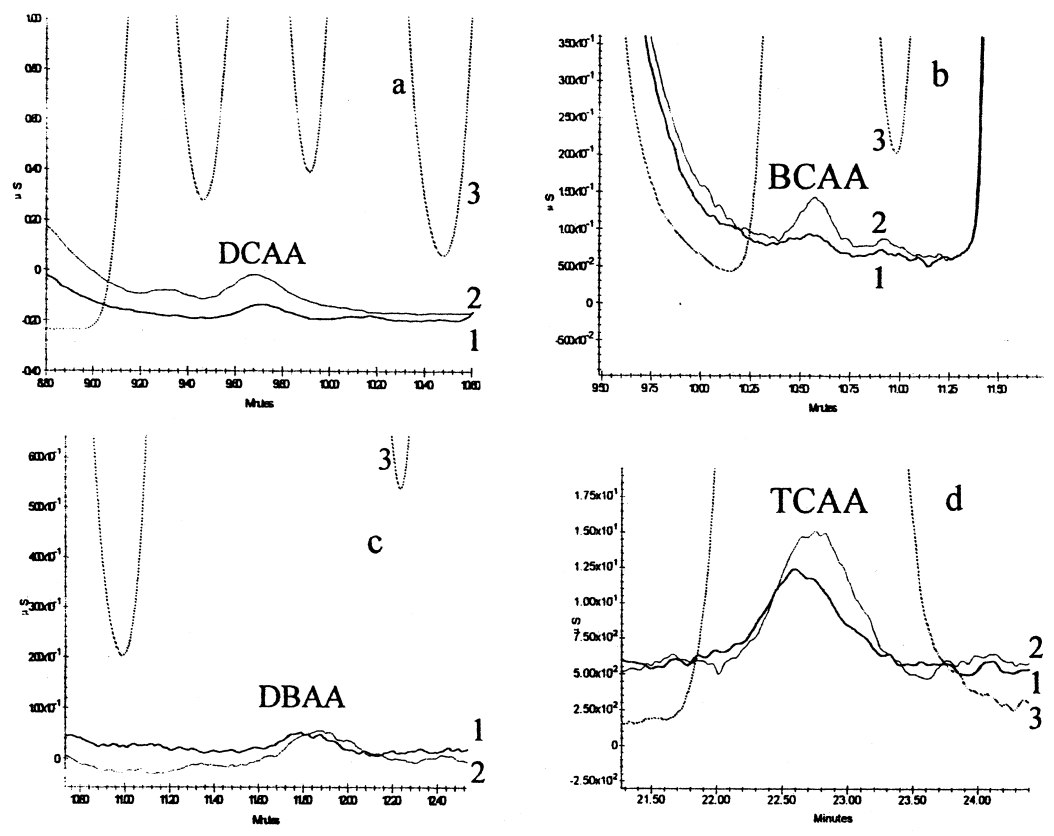

Fig. 3. The chromatograms of the HAAs in real samples with the AS9HC column and ASRS-Ultra suppressor. Dionex IonPac AG9HC and AS9HC columns; detection: suppressed conductivity with ASRS-ULTRA $4 \mathrm{~mm}$ operated at $300 \mathrm{~mA}$ in the autosuppression recycle mode; injection volume: $500 \mu \mathrm{l}$; eluent: $28 \mathrm{mM} \mathrm{Na} \mathrm{CO}_{3}$; flow-rate: $1.0 \mathrm{ml} / \mathrm{min}$ from $0 \mathrm{~min}$ to $15 \mathrm{~min}, 1.5 \mathrm{ml} / \mathrm{min}$ from $15.1 \mathrm{~min}$ to $35 \mathrm{~min}$, $1.0 \mathrm{ml} / \mathrm{min}$ from $35.1 \mathrm{~min} .1=$ Real sample, $2=$ spiked sample, $3=$ standard sample,. 


\subsection{Application of the developed methods to real} drinking water samples

The developed methods were applied to the determination of the nine HAAs and perchlorate in the finished drinking water samples of Beijing's seven drinking water plants. From Fig. 3, it can be seen that DCAA (a), BCAA (b), DBAA (c) and TCAA (d) could be detected with the AS9HC column and ASRS-Ultra suppressor. Because the sensitivity of the method is relatively low, the responses of the analytes were very small. With the AS16 column, in addition to the four analytes, perchlorate (d) and BDCAA were also determined (Fig. 4, Table 3). In some real samples, the concentration of nitrate was so high that it interfered with the determination of DCAA (Fig. 4a). However, the problem could be easily solved by diluting the sample, for the high sensitivity of the method, the diluted DCAA still had significant response. The concentrations of the ana- lytes in the samples of the seven water plants are listed in Table 3. From the table we knew that for the high sensitivity of the AS16 column, more HAAs could be detected. Perchlorate could also be detected in some drinking water samples with the method. With regard to the results obtained with the three methods, all the data were almost the same.

\section{Conclusion}

Three methods were developed for the determination of nine HAAs, perchlorate and some common anions in drinking water. With the AS9HC column and carbonate as eluent, HAAs and six common anions could be determined in one injection. The detection limits for the HAAs were between 1.53 and $53.6 \mu \mathrm{g} / 1$ with the ASRS-Ultra suppressor and from 0.37 to $31.64 \mu \mathrm{g} / 1$ with the anion Atlas electrolytic
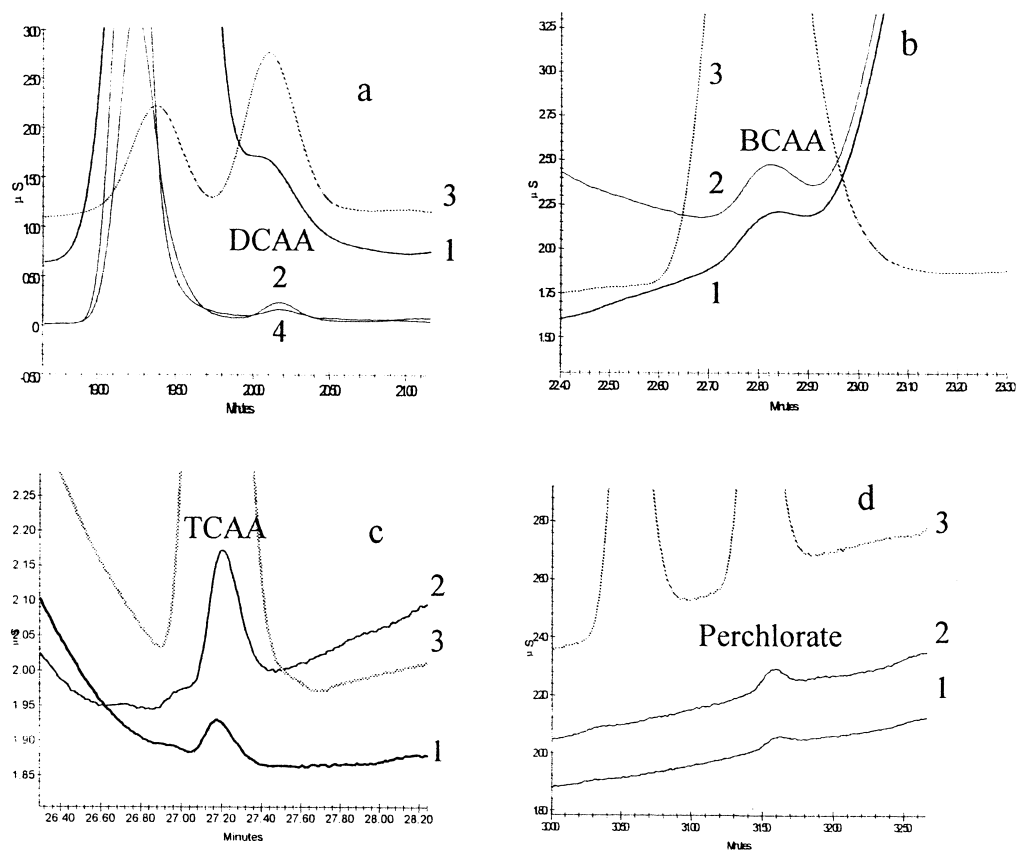

Fig. 4. The chromatograms of the HAAs and perchlorate in real samples on the AS16 column. Dionex IonPac AG16 and AS16 columns; detection: suppressed conductivity with ASRS-ULTRA $4 \mathrm{~mm}$ operated at $300 \mathrm{~mA}$ in the external water mode; injection volume: $500 \mu \mathrm{l}$; eluent: $\mathrm{NaOH}$ in gradient, $4.9 \mathrm{~m} M$ for $9 \mathrm{~min}, 4.9-5.2 \mathrm{~m} M$ in $3 \mathrm{~min}, 5.2-7.2 \mathrm{~m} M$ in $5 \mathrm{~min}, 7.2-30.9 \mathrm{~m} M$ in $3 \mathrm{~min}, 30.9-72.5 \mathrm{~m} M$ in $9 \mathrm{~min}$, $72.5 \mathrm{~m} M$ for $4 \mathrm{~min}$, followed by a linear gradient to $4.9 \mathrm{~m} M$ in $7 \mathrm{~min}$, the content of $10 \%$ methanol solution was $5 \%$ in the eluent all along; flow-rate: $1.0 \mathrm{ml} / \mathrm{min}$. 1 =Real sample, $2=$ spiked sample, $3=$ standard sample, $4=$ diluted sample. 
Table 3

The concentrations and spiked recoveries of nine HAAs and perchlorate in the drinking water samples of the seven water plants with the three methods $(\mu \mathrm{g} / 1)$

\begin{tabular}{|c|c|c|c|c|c|c|c|c|c|c|}
\hline & MCAA & MBAA & DCAA & BCAA & DBAA & TCAA & BDCAA & DBCAA & Perchlorate & TBAA \\
\hline \multicolumn{11}{|l|}{ AS9HC-ASRS-Ultra } \\
\hline$A^{*}$ & - & - & 30.2 & 9.4 & - & - & - & - & - & - \\
\hline B & - & - & 19.3 & & 44.3 & 10.1 & - & - & - & - \\
\hline $\mathrm{C}$ & - & - & - & - & 28.0 & - & - & - & - & - \\
\hline $\mathrm{D}$ & - & - & 36.3 & 20.4 & - & 15.4 & - & - & - & - \\
\hline $\mathrm{E}$ & - & - & 11.4 & 19.3 & 81.3 & - & - & - & - & - \\
\hline $\mathrm{F}$ & - & - & 15.2 & 15.4 & - & 15.3 & - & - & - & - \\
\hline G & - & - & 44.4 & - & - & 38.3 & - & - & - & - \\
\hline $\begin{array}{l}\text { Spiked recovery } \\
\text { of sample B }(\%)\end{array}$ & 96.7 & 102.2 & 103.9 & 100.4 & 98.1 & 97.8 & 103.5 & 99.4 & - & 98.1 \\
\hline \multicolumn{11}{|l|}{ AS9HC-Atlas } \\
\hline A & - & - & 24.1 & 13.1 & 15.1 & - & - & - & - & - \\
\hline B & - & - & 20.2 & 19.0 & 50.0 & 16.0 & 25.1 & - & - & - \\
\hline $\mathrm{C}$ & - & - & 8.4 & 12.1 & 31.0 & - & 29.4 & - & - & - \\
\hline $\mathrm{D}$ & - & - & 35.1 & 21.2 & 15.3 & 33.3 & - & - & - & - \\
\hline $\mathrm{E}$ & - & - & 15.0 & 24.7 & 69.5 & 14.4 & - & - & - & - \\
\hline $\mathrm{F}$ & - & - & 14.3 & 14.3 & 18.3 & 12.4 & - & - & - & - \\
\hline G & - & - & 60.1 & - & 3.1 & 39.2 & - & - & -- & - \\
\hline $\begin{array}{l}\text { Spiked recovery } \\
\text { of sample B }(\%)\end{array}$ & 99.3 & 102.4 & 97.1 & 98.6 & 99.1 & 97.7 & 101.9 & 102.2 & 96.5 & 103.4 \\
\hline \multicolumn{11}{|l|}{ AS16 } \\
\hline A & - & - & 21.3 & 10.2 & 12.1 & 6.6 & 19.3 & - & 4.6 & - \\
\hline B & - & - & 15.5 & 16.3 & 44.1 & 12.3 & 35.0 & - & 2.6 & - \\
\hline $\mathrm{C}$ & - & - & 8.1 & 9.1 & 33.2 & 8.4 & 21.1 & - & 1.5 & - \\
\hline $\mathrm{D}$ & - & - & 33.4 & 18.2 & 13.5 & 23.0 & 12.2 & - & - & - \\
\hline $\mathrm{E}$ & - & - & 13.4 & 26.1 & 78.4 & 10.3 & 22.4 & - & - & - \\
\hline $\mathrm{F}$ & - & - & 11.1 & 14.5 & 22.3 & 17.4 & 7.6 & - & - & - \\
\hline G & - & - & 55.2 & - & 2.1 & 46.4 & - & - & - & - \\
\hline $\begin{array}{l}\text { Spiked recovery } \\
\text { of sample B }(\%)\end{array}$ & 98.8 & 99.1 & 98.5 & 97.9 & 98.0 & 103.2 & 96.7 & 102.6 & 101.9 & 97.8 \\
\hline
\end{tabular}

* A, B, C, D, E, F, G are the seven water plants.

suppressor. By employing the AS16 column, after pretreatment with the OnGuard Ag cartridge, HAAs, perchlorate and three common anions could also be analyzed in $34 \mathrm{~min}$ with gradient of sodium hydroxide as eluent. The method detection limits for the nine HAAs were between 1.11 and $9.32 \mu \mathrm{g} / \mathrm{l}$, and $0.6 \mu \mathrm{g} / 1$ for perchlorate. The developed methods were applied to the determination of the compounds in the finished drinking water of seven water plants in Beijing. The results indicated that DCAA, BCAA, DBAA, TCAA and perchlorate could be detected in the real samples. In the nine HAAs, DCAA and DBAA were the major compounds.

\section{References}

[1] E.W. Akin, J.C. Hoff, E.C. Lippy, Environ. Health Perspect. 46 (1982) 7.

[2] R.F. Christman, D.L. Norwood, D.S. Milington, J.D. Johnson, A.A. Stevens, Environ. Sci. Technol. 17 (1983) 625.

[3] J.D. Johnson, R.F. Christman, D.L. Norwood, D.S. Milington, Environ. Health Perspect. 46 (1982) 63.

[4] A.M. Richare, E.S. Hunter, Teratology 53 (1996) 352.

[5] R.E. Linder, G.R. Klinefelter, L.F. Strader, J.D. Suarez, C.J. Duer, Fundam. Appl. Toxicol. 22 (1994) 422.

[6] R.E. Klinefelter, J.D. Suarez, N.L. Roberts, A.B. Deangelo, Reprod. Toxicol. 9 (1995) 571.

[7] E.T. Urbansky, M.R. Schock, J. Environ. Manage. 56 (1999) 79. 
[8] R.W. Gullick, M.W. Lechevallier, T.S. Barhorst, J. Am. Water Works Assoc. 93 (2001) 66.

[9] Fed. Reg. 63 (1998) 10274.

[10] US EPA, Drinking Water Contaminant List, EPA Document No. 815-F-98-002, GPO, Washington, DC, 1998.

[11] Correspondence from Joan S. Dollarhide, National Center for Environmental Assessment, Office of Research and Development, to Mike Girrard, Chairman, Perchlorate Study Group, US EPA, 1995.

[12] E.T. Urbansky, T.W. Collette, W.P. Robarge, W.L. Hall, J.M. Skillen, P.F. Kane, Survey of Fertilizers and Related Materials for Perchlorate (ClO4-), EPA/600/R-01/049, Environmental Protection Agency, US Government Printing Office, Washington, DC, 2001.

[13] Overview of California's Perchlorate Experience, California Department of Health Services, updated 19 November 1999, http://www.dhs.cahwnet.gov/org/ps/ddwem/chemicals/ perchl/perchl_overview.htm.

[14] J.W. Hodgeson, J. Collins, R.E. Barth, Determination of Haloacetic Acids in Drinking Water by Liquid-Liquid Extraction, Derivatization and Gas Chromatography With Electron Capture Detection, EPA Method 552.0, Revision 1, Methods for the Determination of Organic Compounds, Supplement II, EPA Document No. 600-4-88-039, GPO, Washington, DC, 1990.

[15] D.J. Munch, J.W. Munch, A.M. Pawlecki, Determination of Haloacetic Acids and Dalapon in Drinking Water by LiquidLiquid Extraction, Derivatization and Gas Chromatography With Electron Capture Detections, EPA Method 552.2, Revision 1, Methods for the Determination of Organic Compounds, Supplement III, EPA Document No. 600-R-95131, GPO, Washington, DC, 1995.

[16] B. Ells, D.A. Barnett, K. Froese, R.W. Purves, S. Hrudey, R. Guevremont, Anal. Chem. 71 (1999) 4747.

[17] B. Ells, D.A. Barnett, R.W. Purves, R. Guevremont, Anal. Chem. 72 (2000) 4555.
[18] M.L. Magnuson, C.A. Kelty, Anal. Chem. 72 (2000) 2308.

[19] S. Hashimoto, A. Otsuki, J. High Resolut. Chromatogr. 21 (1998) 55.

[20] H. Carrero, J.F. Rusling, Talanta 48 (1999) 711.

[21] D. Martínez, J. Farré, F. Borrull, M. Calull, J. Ruana, A. Colom, J. Chromatogr. A 808 (1998) 229.

[22] D. Martínez, F. Borrull, M. Calull, J. Chromatogr. A 835 (1999) 187.

[23] C. Sarzanini, M.C. Bruzzoniti, E. Mentasti, J. Chromatogr. A 850 (1999) 197.

[24] P. Akhtar, C.O. Too, G.G. Wallace, Anal. Chim. Acta 341 (1997) 141.

[25] L.M. Nair, R. Sarri-Nordhaus, J.M. Andersor Jr., J. Chromatogr. A 671 (1994) 309.

[26] Y. Lopez-Avila, Y. Liu, C. Charan, J. AOAC Int. 82 (1999) 689.

[27] C.J. Koester, H.R. Beller, R.U. Halden, Environ. Sci. Technol. 34 (2000) 1862.

[28] M.L. Magnuson, E.T. Urbansky, C.A. Kelty, Anal. Chem. 72 (2000) 25.

[29] Y.J. Liu, S.F. Mou, S. Herbling, J. Chromatogr. A 956 (2002) 85.

[30] P.E. Jackson, M. Laikhtman, J.S. Rohrer, J. Chromatogr. A 850 (1999) 131.

[31] R. Stahl, Chromatographia 37 (1993) 300.

[32] C.G. Neuhold, K. Kalcher, X. Cai, G. Raber, Anal. Lett. 29 (1996) 1685.

[33] L.K. Jackson, R.J. Joyce, M. Laikhtman, P.E. Jackson, J. Chromatogr. A 829 (1998) 187.

[34] J.R. Stillian, C.A. Pohl, J. Chromatogr. 499 (1990) 249.

[35] H. Small, in: Ion Chromatography, Plenum Press, New York, 1989 , p. 37.

[36] Installation Instructions and Troubleshooting Guide for the OnGuard II Cartridges, Document No. 031688, Revision 01, 28 March 2000, Dionex, Sunnyvale, CA, 2000. 